\title{
Examining student attitudes to improve an undergraduate online engineering course
}

Mr. Devayan Debashis Bir, Iowa State University

Dr. Benjamin Ahn, Iowa State University 


\title{
Examining student attitudes to improve an undergraduate online engineering course
}

\begin{abstract}
Due to the advent of technology and the world wide web, online engineering courses have gained prominence and have become a popular way to learn new content. Universities around the country, in addition to traditional face-to-face courses, are taking advantage of technology and the web to offer online courses to their engineering students. Despite the sudden increase in online courses, students' attitudes toward these courses remain unknown. This study examined students' attitudes towards an online engineering course, specifically determining what students found most and least helpful when learning online, what students liked and disliked about the course, and what they perceived were the advantages and disadvantages of taking the online course.
\end{abstract}

The study examined an engineering course titled, Mechanics of Materials, an introductory mechanics course for sophomore engineering students from Aerospace, Mechanics, Civil and Construction, and other engineering disciplines. Historically, the course has been taught in a traditional lecture class, but this study examined a section taught online. Findings show that students liked the availability of the lecture and sample problem videos and liked pacing their learning to their own needs.

\section{Introduction and Literature Review}

Continuous developments in web technology and the use of online teaching mediums, such as Blackboard, have made it easier to implement online courses that are now offered at universities around the United States and are becoming increasingly popular due to the schedule flexibility for students, availability of a wide variety of course materials, integrated clips that offer an effective delivery method for teaching, and the possibility of a reduced degree cost. With their increasing popularity, it is important to provide quality education through these mediums by a continuous effort to improve the course content and delivery. One way to do so is to take into account student attitudes towards online courses to improve the content provided and to determine which aspects of the course to focus on.

Undergraduate online engineering courses (UOECs) are offered to both working professionals and full time students at many universities in the United States. Since these classes count towards a student's graduation or certification, it is of utmost importance to keep improving these classes to provide better education. A study by the U.S. Department of Education suggested that the academic performance of online students is "modestly better" than face-to-face traditionally taught students [1]. Furthermore, according to a study [7] that surveyed 45 randomly selected U.S. based institutions, online learning offers new avenues and challenges to educational instruction. The study urged further research not only in providing online educational opportunities but also in achieving the highest educational quality through this medium.

Although online classes have shown promise in other fields of education, limited studies have explored their effects in engineering education. A study [9] conducted in 2005 made a strong 
argument for the use of online courses for engineering education. It predicted that if online education was to become widespread, the trend of seeing online tools to teach blended classes would become popular. It also predicted that the quality of online course would improve through interactive teaching mediums and constructivist methodologies. The article recommended that engineering colleges explore new methodologies plausible within the online medium that are best applicable to engineering education. In as much as numerous studies have shown the importance of evaluating student perceptions of online courses to tailor the course according to learner needs [2, 3, 4, 8, 10], this study examined students' attitudes towards an online engineering course to evaluate the effectiveness of various aspects of the course content and its delivery.

The UOEC under scrutiny in this paper was a Mechanics of Materials course offered in the Aerospace Engineering Department at Iowa State University. The course consisted of weekly modules, which were inclusive of topic video lectures, instructor-solved sample problem videos, course materials (e.g., lecture slides, supplemental readings), and assignments. The assignments were to be submitted by the students at the end of each week. Each module included videos consisting of three to four topics introducing new concepts and solved sample problems. Each topic video was approximately 12 minutes long, and the sample problem videos were 10 minutes. During the semester, students could contact the professor via email regarding questions or use the online office hours where the professor and students could virtually meet. The faculty who taught the course created the lecture videos of the UOEC.

The specific question and comments this study attempted to examine from students taking the online Mechanics of Materials course were:

- Describe the course activities that are most helpful and least helpful to your learning in this course.

- What do you like and dislike about the course?

- List the advantages and disadvantages of the online course compared to traditional courses.

Methods

Settings and Participants

This research was conducted at Iowa State University (ISU) and the participants were undergraduate engineering students who had enrolled for the online Mechanics of Materials course in either summer 2016 or spring 2017. Mechanics of Materials is a required course for engineering major students from aerospace, mechanical, and civil and construction engineering departments at ISU. All students had an option to enroll in either an online or a traditionally taught face-to-face course. Mechanics of Materials was offered as an online course for the first time in summer 2016 at ISU. The summer 2016 cohort consisted of 40 students and was conducted over a 10-week period. The spring 2017 cohort consisted of 30 students and was conducted over a 15-week period.

\section{Research Design}

The research findings presented in this paper are from a cohort of students enrolled in the 
summer 2016 course and who completed the survey. An anonymous survey was administered in the last week of the summer 2016 semester to examine students' attitudes towards the course. The students were given \$10 gift cards and five points on their final score to incentivize them. The survey consisted of questions about the students' attitudes towards the various aspects of the course and how it compared with the traditional face-to-face courses they had taken previously. The investigators of the study analyzed these qualitative data obtained from the anonymous survey. Each response to the open-ended questions on the survey was analyzed for common patterns and trends, which were then made into theme categories. The survey also included questions on demographic information such as enrollment status (part-time/full-time), gender, major, ethnic background, and nationality (U.S. citizen/International). The survey additionally asked Likert type questions about course content such as appropriate length, quantity, quality, and if it supported students' learning.

To improve the reliability, each question on the survey was examined by two qualitative analysis experts for biases. Once the biases had been removed, consent from the study participants was obtained. Each student enrolled for the course was sent a consent letter that described the study and the motivation behind it. The letter also stated that the responses from the survey were anonymous so that the participants could answer the survey questions freely and to the best of their knowledge. The survey had a response rate of $88 \%$ (35 out of 40 students).

\section{Data Analysis}

The summer survey data were downloaded from the Qualtrics database. The survey responses were then analyzed to generate common themes for each question. Each response was then put into one of the themes. A codebook (listed in Table 1) was made that listed all the themes generated in the survey analysis. The distribution of the responses for each question showed the students attitudes towards the course aspect or course content. To protect the confidentiality of the students, data were presented so that no student could be identified.

The items that emerged from the survey administered to the summer 2016 students are listed in Table 1.

Table 1. Theme definitions and sample student quotes

\section{Lecture Videos (LV)}

- Student responses about the lecture videos provided to them through the online module on Blackboard.

"The lectures are easily the most helpful. I read the book first, and sometimes try the homework before watching the videos.

"Lecture videos are the least helpful for me because my learning style is more focused on working problems rather than being told how to solve a problem."

\section{Notes (N)}

- Student responses that relate to the use of various materials provided through Blackboard. The materials include the e-book, slides and practice problems.

"The least helpful activity is just viewing the slides without the video because the slides don't always show all of the written information."

"The book readings are least helpful. I get a general understanding of the topic, but it isn't 
until the lecturers and example problems that the topic makes sense."

\section{Example Videos (EV)}

- Student responses about the example videos provided to them through the online module on blackboard. The example videos show the students how to solve a numerical problem systematically.

"Example videos are very helpful, and the instructor's additional comments pertaining to various details..."

"The example videos where problems are worked through seem to help me grasp concepts."

\section{Homework (HW)}

- Student responses about the weekly homework assigned to them.

"The homework is the most helpful."

\section{Exams and Tests (ET)}

- Student responses about the exams and practice tests, which had to be taken to complete the course.

"I would say the practice test give you the best idea of what to expect on the test."

\section{None (NN)}

- Student responses which stated "none".

\section{Forums (F)}

- Student responses to the use of discussion boards available on Blackboard.

"There aren't a ton of components but sometimes the help forum doesn't work for me. It's harder to understand the questions and explanations without visuals."

\section{Grading Scheme (GS)}

- Student responses on the assigning of marks for exams and homework and how they were graded.

"The class is almost all test grades. More of the class should be gauging how much work you put into it. Homework should be worth more than 10\% for how much time it takes and how well it helps you understand the material."

\section{Time-Management (TM)}

- Student responses about managing their time to finish the course which included lecture videos, course materials, and homework.

"It's easy to get behind on coursework. I also had very limited amount of time to study for exams or complete assignments because I was working full time."

\section{Course Flexibility (CF)}

- Student responses about being able to take the course without being physically present in class by doing the online module and exams at a convenient time.

"Can do it on your own time so your work schedule is unaffected, don't have to worry about attending class, all materials are easily accessible."

"Working according to your own schedule."

\section{Pace of Studying (PS)}

- Student responses about being able to go through the online module at their own speed so that they understand the concepts.

"Some advantages are that you can work on your own time to learn the material."

\section{Face-to-Face Interaction (FFI)}

- Student responses related to not being able to physically communicate with the faculty and 
other students.

"Cannot face-to-face ask questions instantly; limited source, no friends for face-to-face discussion."

Results

This section provides the results to the three examined questions. The total of 35 students responded to the survey.

Q1: Describe the course activities that are most and least helpful to your learning in the course. Table 2 shows the distribution of responses given by students for activities that were most and least helpful in their learning. For example, 12 students responded that homework was the activity most helpful to their learning. Out of those 12 students, three, one, five, and three students considered exams and tests, forums, lecture videos, and no aspect of the online course, respectively, to be least helpful to their learning.

Table 2. Cross tabulation of survey responses for the questions towards course activities

\begin{tabular}{cccccccc}
\hline $\begin{array}{c}\text { Activities } \\
\text { most } \\
\text { Helpful }\end{array}$ & ET & F & HW & LV & N & NN & Total \\
\hline ET & 0 & 1 & 2 & 1 & 0 & 0 & 4 \\
\hline EV & 0 & 0 & 0 & 3 & 1 & 1 & 5 \\
\hline HW & 3 & 1 & 0 & 5 & 0 & 3 & 12 \\
\hline LV & 2 & 2 & 6 & 0 & 2 & 1 & 13 \\
\hline N & 0 & 0 & 1 & 0 & 0 & 0 & 1 \\
\hline Total & 5 & 4 & 9 & 9 & 3 & 5 & 35 \\
\hline
\end{tabular}

From the above table we can see that lecture videos $(n=13)$ and homework $(n=12)$ were the activities most helpful to students. Somewhat ironically, lecture videos $(\mathrm{n}=9)$ and homework $(n=9)$ were also the activities least helpful to students. This shows that students have a clear preference to learn from either one of these activities.

Students who considered lecture videos the most helpful activity to their learning said they liked the fact that they were able to pause the videos and take notes. A student responded, "Learning the course from the video; video playback is the most helpful because I get to watch it repeatedly till I understand." The negative aspects regarding the lecture videos were that they were either too short (according to one of the students, "The lectures are way too short and do not help at all learning. Feels like I am learning all by myself half of the time"). An interesting finding here is that although students had mixed attitudes towards the lecture videos, they responded about the example videos $(\mathrm{n}=5)$ only with positive attitudes, primarily because they were able to understand how to solve problems through the steps shown in the example videos. A student responded, "The example videos where problems are worked through seem to help me grasp concepts." Students considered homework the most helpful activity to their learning primarily because it allowed them to practice numerical problems and prepared them for exams. A student responded, "The homework and practice problems that are done during the lectures are the most helpful. I learn much better with examples." 
Q2: What do you like and dislike about the course?

Table 3 shows the distribution of responses given by students on the aspects of course that they liked or disliked. Apart from lecture videos $(\mathrm{n}=6)$ and notes $(\mathrm{n}=5)$, the course aspects liked by the students were course flexibility $(n=16)$ and pace of studying $(n=6)$. Both of these aspects are well known and intuitive advantages of taking online courses. For these students, the biggest advantage of taking the online course was course flexibility because they could dictate their own schedules. A typical response was "I like the freedom of the course to manage your time on your own rather than at specific times for lectures."

Table 3. Cross tabulation of survey responses for the questions towards course aspects

\begin{tabular}{|c|c|c|c|c|c|c|c|c|}
\hline \multirow{2}{*}{$\begin{array}{c}\text { Course } \\
\text { likes }\end{array}$} & \multirow[b]{2}{*}{ ET } & \multirow[b]{2}{*}{ FFI } & \multicolumn{6}{|c|}{ Course dislikes } \\
\hline & & & G & HW & LV & $\mathbf{N}$ & TM & Total \\
\hline CF & 4 & 1 & 1 & 3 & 1 & 1 & 5 & 16 \\
\hline EV & 0 & 0 & 0 & 0 & 1 & 0 & 0 & 1 \\
\hline $\mathbf{L V}$ & 2 & 0 & 1 & 2 & 0 & 0 & 1 & 6 \\
\hline $\mathbf{N}$ & 0 & 0 & 0 & 2 & 1 & 0 & 0 & 5 \\
\hline $\mathbf{N N}$ & 0 & 0 & 0 & 0 & 1 & 0 & 0 & 1 \\
\hline PS & 2 & 1 & 1 & 1 & 1 & 0 & 0 & 6 \\
\hline Total & 8 & 2 & 3 & 8 & 5 & 1 & 8 & 35 \\
\hline
\end{tabular}

Students had negative attitudes towards the homework $(\mathrm{n}=8)$, the exams and tests $(\mathrm{n}=8)$, and their time-management $(\mathrm{n}=8)$ during the course. The students disliked the exams and tests primarily because while they could determine their own schedules with regard to the online modules, they had predetermined exam times to which they had to adhere. Another point to note here is that although students enjoyed the flexibility $(n=16)$ the online course offered, five of these students were unable to manage their time to finish the course modules up to their own expectations. The course was designed in modules, intended to make the online learners more independent, yet a student disclosed, "It is pretty easy to get behind on the coursework. I also had very limited time to study for exams or complete assignments because I was working full time." This student's response supports previous findings that self-regulation is one of the most critical factors that influence the learning outcome of students in online courses [11].

Q3: List the advantages and disadvantages of the online course as compared to traditional courses.

Table 4 shows the distribution of responses given by students on the advantages and disadvantages of the online course. For example, 20 students responded that the flexibility of the course to be one of the advantages of the online course, however, 14 of those students stated that they missed face-to-face interactions with faculty to be a disadvantage of the online course.

Table 4. Cross tabulation of survey responses for the questions towards course advantages and disadvantages

\begin{tabular}{ccccc}
\hline Course & \multicolumn{5}{c}{ Course disadvantages } \\
\cline { 2 - 5 } advantages & FFI & N & TM & Total \\
\hline CF & 14 & 2 & 4 & 20 \\
\hline
\end{tabular}




\begin{tabular}{ccccc}
\hline NN & 1 & 1 & 0 & 2 \\
\hline PS & 11 & 1 & 1 & 13 \\
\hline Total & 26 & 4 & 5 & 35 \\
\hline
\end{tabular}

The biggest challenge faced by students in the online course was lack of face-to-face interaction $(\mathrm{n}=26)$. Students missed not being able to meet with other students and the professor, even though they could meet the professor virtually online. A student expressed, "Cannot face-to-face ask questions instantly; limited source, no friends for face-to-face discussion." Even though timely feedback was provided to the students, they still felt a lack of interaction. Again, the analysis showed that among the students who responded with course flexibility as an advantage, four of these students responded with time-management as a disadvantage. A response revealing the attitudes of these students was "I'm more likely to wait till the last minute to do everything."

The results imply that students felt strongly either positively or negatively about lecture videos, notes, homework, and exams and tests. These aspects of the course could be modified to help students learn better and become self-directed learners. Overwhelmingly, students missed faceto-face interaction with other students and the faculty. This aspect of the online course needs modification, possibly including teamwork exercises.

\section{Discussion}

The above findings on the students' perceptions towards the online course suggest that the course needs to be designed for students to become more independent learners so that they can manage their time to finish the course modules and feel satisfied with the learning. The results also point towards implementing collaborative efforts so that the students interact with each other, which could substitute for the lack of face-to-face interaction.

It is still not known what kind of lecture videos, notes, homework, and exams applicable to the online course would not only keep the students interested and satisfied with the course but also improve the learning process. A study [9] that used a video-game-based approach to teach an online engineering course shows promise. This approach kept the students more engaged with the course and promoted a deeper student learning of the concepts when compared to a traditional course. It also improved the students' perception of the importance of the course. The video-game-based course was rated as one of the most important courses while the traditional course was rated as one of the least important. The game-based approach was able to transform the course from exams- and grades-oriented to one more applicable in the real world. The study suggested further research into activities that could have the same effect as the game-based approach. The key to creating an online course is to change the epistemological statement of the course to one that promotes professional and readily applicable learning instead of the traditional grades and exams.

Suggestions for a revised undergraduate online engineering courses

To improve the quality of learning in the UOEC, collaboration between students will be encouraged. To implement collaboration in the UOEC the following additions could be made.

- Discussion on weekly reports: A project report will be posted on the discussion forums for the students to read and discuss with other students to exchange ideas. 
- Group projects: The class will be divided into groups of three. These groups will submit a short paper on a research idea and its applications from the concepts learned through the course.

For example, a weekly report will have students complete simulation tutorials on cantilever beams with various types of loadings, such as uniformly distributed loads and point loads. These simulations can be performed using software like Solidworks, ANSYS or Abaqus. The tutorial will encompass all the basics (i.e. geometry creation, meshing, and solver selection) necessary to perform such simulations. Since the course is offered online, students have the choice of either downloading a student version of the simulation software or using the software available on campus via Virtual Desktop. Completing the tutorials will better equip student to understand how the deflections on beams occur due to applied loads. The students will get an opportunity to discuss the weekly reports with the TA and other students in the course over online course forums.

As for the group project, after the first half of the semester, the student groups, which will be predefined, will have to come up with demonstrations/experiments that apply some components of what was covered in the course for their final projects. These projects must demonstrate the group's learning of mechanics of materials' concepts and their applications. The point of this exercise is to promote the effective use of forums and team collaboration in the course.

These weekly reports and group projects will be implemented for students enrolled in future semesters. The students will be asked to complete a survey that will ask open-ended, Likert type questions about these additions and will examine the student attitudes towards a course designed completely in the above-mentioned manner. It is important to know the issues and challenges faced by students taking the online course so that the course can be tailored to their needs. The course should be able to satisfy the expectations of its learners while, at the same time, providing them with a quality education. To this end, it is essential to discover the attitudes and perception of students towards the course content and other various aspects of the course.

In the study reported here, a few factors may have affected the students' behavior and attitudes toward the course. First, since the course was offered during the summer semester, most participants were either working or on an internship. This could have caused the students to not be able to manage their time or devote enough time to the course. Second, students had a choice of which pedagogy (i.e., online or traditionally taught face-to-face) to enroll in. The fact that students decided to select the online version of the course may have caused self-selection bias towards online courses.

The findings of the study throw light on the aspects of the course that need improvement. Students need to discuss and negotiate their ideas and collaborate to solve problems. These help in the learning process and are in line with social constructivist perspectives [5]. The issues students face in this online course would also translate to other UOECS, and the inferences made from this study would be applicable to other UOECs.

Future Work 
The same survey used in summer 2016 will again be administered to students taking the course in spring 2017 in order to examine students' attitudes towards the online course and various aspects of the course. Additionally, one-on-one interviews will be conducted with each of the participants of the study. The interview questions will ask about student experiences of the UOEC, tactics employed by the students in completing the various aspects of the course, and suggestions to improve the course. Each interview is expected to last for approximately 30 minutes. These interviews will be scheduled in the final week of the semester. The students will be given $\$ 10$ gift cards for completing the survey and participating in the interviews.

The survey and interviews will be analyzed in a manner similar to summer 2016. Each question response will be categorized according to the codebook developed previously. New themes will be generated for the responses that do not fit within the themes defined in the codebook. The personal interviews will be audio recorded so that they can be transcribed verbatim. A matrix will be built for each question consisting of the themes and corresponding responses from all the transcripts.

The qualitative data obtained from surveys and personal interviews will be analyzed to answer additional research questions:

- What are the students' habits regarding completing the various course aspects?

- What modifications can be made to the UOEC to improve student learning?

\section{References}

1. Means, B., Toyama, Y., Murphy, R., Bakia, M., \& Jones, K. (2009). Evaluation of evidencebased practices in online learning: A meta-analysis and review of online learning studies. US Department of Education.

2. Ruey, S. (2010). A case study of constructivist instructional strategies for adult online learning. British Journal of Educational Technology, 41(5), 706-720.

3. Maor, D., \& Fraser, B. J. (2005). An online questionnaire for evaluating students' and teachers' perceptions of constructivist multimedia learning environments. Research in Science Education, 35(2), 221-244.

4. Rovai, A. P. (2004). A constructivist approach to online college learning. The internet and higher Education, 7(2), 79-93.

5. Palincsar, A. S. (1998). Social constructivist perspectives on teaching and learning. Annual review of psychology, 49(1), 345-375.

6. Coller, B. D., \& Scott, M. J. (2009). Effectiveness of using a video game to teach a course in mechanical engineering. Computers \& Education, 53(3), 900-912.

7. Chen, P. S. D., Lambert, A. D., \& Guidry, K. R. (2010). Engaging online learners: The impact of Web-based learning technology on college student engagement. Computers \& Education, 54(4), 1222-1232.

8. Branoff, T., \& Wiebe, E. (2009). Face-to-face, hybrid, or online?: Issues faculty face redesigning an introductory engineering graphics course. Engineering Design Graphics Journal, 73(1).

9. Bourne, J., Harris, D., \& Mayadas, F. (2005). Online engineering education: Learning anywhere, anytime. Journal of Engineering Education, 94(1), 131-146. 
10. Bir, D., \& Ahn, B. (2016, October). Applicability of online Mechanics of Materials course for engineering undergraduate students. In Frontiers in Education Conference (FIE), 2016 IEEE (pp. 1-3). IEEE.

11. Steif, P. S., \& Dollár, A. (2009). Study of Usage Patterns and Learning Gains in a Web-based Interactive Static Course. Journal of Engineering Education, 98(4), 321-333.

\section{Appendix}

\section{Sample Interview protocol}

1) What was your motivation for choosing the online version of the course over the traditional face-to-face version of the course?

2) What are your thoughts so far with respect to the course?

3) What are your thoughts on the lecture videos provided in the course?

4) What are your thoughts on the example videos provided in the course?

5) Do you have a preference for a type of video?

6) Can you describe your video viewing habits?

a) Do you watch the videos? How do you watch them?

b) How do you watch a week's worth of topics? Do you watch them all at once?

c) What are the reasons for viewing the video multiple times?

7) What materials have been most helpful in learning the course content?

8) How do you manage your time regarding the coursework?

a) Tell me how you typically study a topic.

b) Tell me how you study over a period of a week.

9) What are the benefits of taking the course online?

10) What challenges have you faced, if any, while taking this online course?

11) What improvements or changes do you suggest for the second half of the semester or for future EM324 XE courses?

12) Describe your satisfaction level with the course. 